\title{
Identification of Relaxation and Diffusion Mechanisms in Amorphous Silicon
}

\author{
G. T. Barkema \\ Institute for Theoretical Physics, Utrecht University, P.O. Box 80000, 3508 TA Utrecht, The Netherlands* \\ Normand Mousseau \\ Computational Physics, Faculty of Applied Physics, Technische Universiteit Delft, Lorentzweg 1, 2628 CJ Delft, The Netherlands, \\ and Department of Physics and Astronomy and CMSS, Ohio University, Athens, Ohio 45701 ${ }^{\dagger}$
}

(Received 28 April 1998)

\begin{abstract}
The dynamics of amorphous silicon at low temperatures can be characterized by a sequence of discrete activated events, through which the topological network is locally reorganized. Using the activation-relaxation technique, we create more than 8000 events, providing an extensive database of relaxation and diffusion mechanisms. The generic properties of these events - size, number of atoms involved, activation energy, etc. - are discussed and found to be compatible with experimental data. We introduce a topological classification of events and apply it to study those events involving only fourfold coordinated atoms. For these, we identify and present in detail three dominant mechanisms. [S0031-9007(98)06947-6]
\end{abstract}

PACS numbers: 61.43.Dq, 82.20.Pm, 82.20.Wt

At low temperatures, the microscopic dynamics of materials typically takes place by activated processes, in which the energy barriers crossed are high compared to the average thermal energy. In crystals, the microscopic mechanisms at the origin of the dynamics can often be found based on symmetry arguments. Disordered materials, however, require direct simulations in order to identify these mechanisms. The standard approach, introduced by Weber and Stillinger [1], is to simulate a material at a high temperature and to quench configurations at regular intervals. If this temperature is sufficiently high and the time between quenches large enough, these configurations will be distinct, and their sequence may provide a basis for the reconstruction of activated dynamics. Difficulties arise, however, since (1) it is never certain that the path reproduced is one that the configuration would follow if simulated at a lower temperature and (2) the Meyer-Neldel compensation rule, affecting the prefactor of the diffusion constant, often favors different mechanisms at high and low temperatures [2].

Using the recently proposed activation-relaxation technique [3], it is possible to follow the reaction path as configurations move from one low-energy structure to another, allowing barriers on all energy scales to be crossed. We apply this method to generate a database of structural changes in amorphous silicon that can then be classified according to their topology, which is well defined since the first- and second-neighbor shells are completely separated in high-quality simulation cells. Besides being an important material in its own right because of its technological importance, amorphous silicon is also the archetypical example of the class of tetrahedrally coordinated continuous-random networks.

Self-diffusion in $c$-Si has an activation energy of about $4.5 \pm 0.5 \mathrm{eV}$ [4]. For $a$-Si, experimentalists find a range of activation energies indicating a multiplicity of mechanisms. In differential calorimetric and conductivity measurements on samples freshly prepared by ion bombardment, the average activation barrier is found to vary from 0.23 to $2.7 \mathrm{eV}$ as a function of relaxation or temperature $[5,6]$. Since samples are far from equilibrium, these barriers have to be taken as lower bounds: barriers should be higher in well-relaxed samples, reaching values similar to those found in $c$-Si.

The nature of relaxation and diffusion mechanisms itself is still controversial: calorimetric and Rahman measurements indicate that relaxation should be local, involving relaxation of point defects [5], while Mössbauer experiments suggest that up to $10^{4}$ atoms are involved, at least to some degree, in the relaxation of a single dangling bond [7].

On the theoretical side, studies of the dynamics of $a$-Si have concentrated so far on very small lattices and have produced only a handful of events. It is therefore difficult to draw solid conclusions from these works, either regarding the activation energies or the mechanisms involved.

In this work, we use the activation-relaxation technique (ART) to generate efficiently a large number of events. ART is described in detail elsewhere [8]. In order to produce good structural amorphous silicon, we proceed as in $[3,9]$, and use the Stillinger-Weber potential [10] with a three-body interaction increased by $50 \%$. After each event, the volume of the cubic cell is optimized to minimize the total energy of the network. For each of three independent runs, we start from a different randomly packed 1000-atom cell relaxed to a local minimum, and apply ART steps iteratively until the configuration reaches a good structural quality $[3,9]$. ART moves that converge to a saddle point within at most 600 iterations are either accepted or rejected, using a Metropolis accept-reject criterion with a fictitious temperature of $0.25 \mathrm{eV}$. The 
optimization phase typically requires a couple of events per atom; just to be sure, we discard the first 4000 ART moves. After optimization, we make another 6000 ART moves, ending with a combined database of 8106 events that are examined here.

The distribution of energy barriers $B$, as well as the energy difference between the initial and final configurations $\Delta E=E_{f}-E_{i}$, is given in Fig. 1 for the whole set of events. The precision in the energy barrier is about $\pm 0.5 \mathrm{eV}$ due to the approximations used in finding the saddle point [8]. The exact shape of these two distributions is a convolution of the topography of the energy landscape with the biases of the activation-relaxation technique [8]; since these biases are not known, one cannot infer quantitative information from the frequency of occurrence of certain events, although we might expect a certain correlation between this and the real frequencies of specific events.

As can be seen in Fig. 1, the distribution for the energy barrier peaks around $4 \mathrm{eV}$ and tails off beyond $15 \mathrm{eV}$. Although the high-energy tail of the distribution is unlikely to be sampled in reality - its time scale goes much beyond what can be reached experimentally - it demonstrates the ease with which ART can explore the energy landscape of disordered materials. The continuous spectrum of energy barriers is in line with experimental data [5]. Looking more closely, the small peak around $0 \mathrm{eV}$ in the distribution of the energy barrier is associated with unstable configurations, and the large peak in the distribution of the energy difference corresponds to atomic exchanges as discussed below. As expected for well-relaxed configurations, most activated mechanisms lead to states of higher energy; only a small proportion of events should lower the energy.

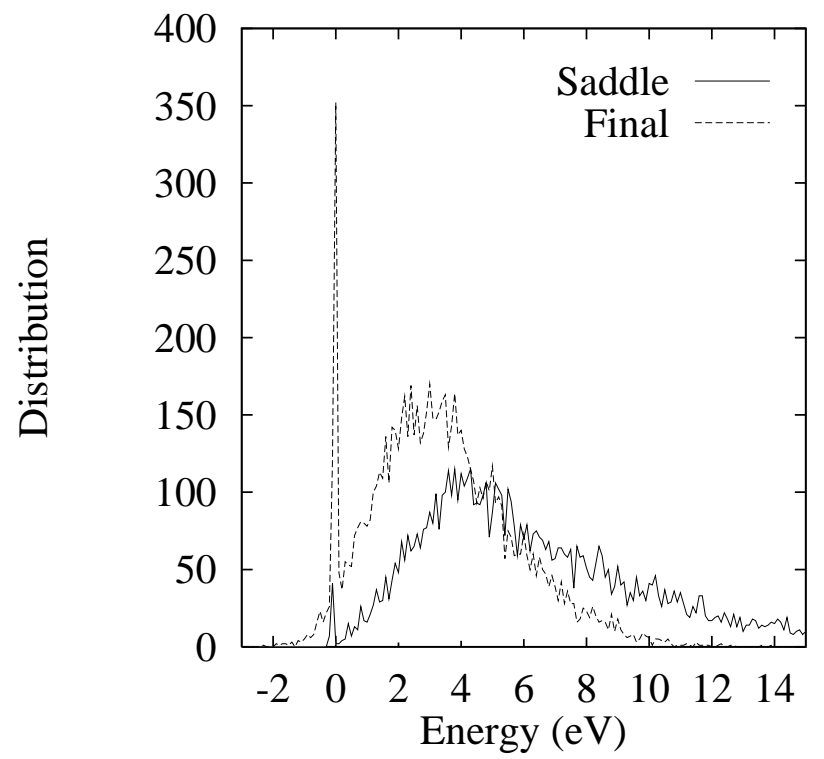

FIG. 1. Distribution of energy barrier $B$ (solid line) and energy difference $\Delta E$ (dotted line) for the 8106 events mentioned in the text.
The size of an event, as defined by the number of atoms that move more than a certain threshold distance, can also provide some insight into the nature of relaxation. With a threshold of $0.1 \AA$, the number of atoms displaced from a minimum to a saddle point varies between 1 and 80, with a peak at about 25. From the initial to the final minimum, this number runs to above 100 with a maximum at 40 . This result sets the minimal system size for the study of these mechanisms to several hundreds of atoms to limit boundary effects, an order of magnitude larger than the typical size of the relaxation mechanism. Moreover, the distribution of the number of atoms involved is almost independent of the specific topological class studied.

Looking at the topology of the structural changes in more detail, we concentrate on two classes of events: perfect events that directly involve only fourfold coordinated atoms, and conserved events that involve the diffusion of coordination defects, while the total number of defects is preserved from the initial to the final state. (By directly involve, we mean atoms that see their neighbor list change between the initial and final state.) There are 802 perfect events, and another 1979 conserved events; the remaining 5325 describe the creation and/or annihilation of coordination defects.

We now consider the 802 perfect events. Table I shows first that, contrary to previous conclusions [11], stretched bonds do not play a major role in the relaxation and diffusion mechanisms of well-relaxed structures. This is in agreement with the narrow spread of nearest-neighbor distances seen experimentally; long bonds are most probably an artifact of incompletely relaxed computer-prepared structures. Table I also indicates that the bond-breaking occurs in a complex process which involves in parallel the formation of new bonding and a considerable local rearrangement. Such a mechanism allows the total amount of energy needed at any time to be much smaller than what a naive evaluation, considering the process of breaking bonds isolated from that of creation, would give.

A topological classification provides more insight into the perfect events. Since the number of broken bonds must be identical to that created between the initial and final events, and all atoms changing neighbors must remain fourfold coordinated, the simplest topological description is to alphabetically label all atoms involved, and list all bonds between them, present before or after the event, or both. Figure 2 shows a typical bond rearrangement.

A more compact classification of the rearrangement is based on the observation that loops of alternating old and new bonds exist that visit all atoms involved (except for the rare cases in which the atoms involved belong to spatially disconnected groups), as was pointed out by Wooten and Weaire [12]. If these atoms are labeled alphabetically, each loop is characterized by the sequence in which atoms are visited. Always using the alphabetically lowest notation guarantees us a nondegenerate topological classification for all perfect events; the event in Fig. 2 is thus labeled "ABACBD." For more complex events, 
TABLE I. Some properties of different mechanisms. $N$ is the number of these events in the database; $b_{i s}$ and $c_{i s}$ refer to the average number of broken and created bonds between the initial minimum and the saddle point; $b_{i f}$ is the same quantity between initial and final minimum, and $l_{i f}^{b}$ and/or $l_{i f}^{c}$ give their respective length in $\AA$; $d$ is the average atomic displacement between initial and final points $(\AA) ; B$ and $\Delta E$ correspond to the maxima in the histograms of the energy barriers and energy changes (eV). The numbers in brackets are the fluctuations in these quantities. The conserved events analyzed here are those with an energy barrier smaller than $8 \mathrm{eV}$.

\begin{tabular}{cccccc}
\hline \hline Type & Perfect & WWW & CE & Double & Conserved \\
\hline$N$ & 802 & 545 & 94 & 64 & 1147 \\
$b_{i s}$ & $3.3(1.6)$ & $2.6(1.2)$ & $5.4(1.3)$ & $4.3(1.3)$ & $3.7(1.4)$ \\
$c_{i s}$ & $2.8(1.5)$ & $2.2(1.0)$ & $4.7(1.6)$ & $3.8(1.5)$ & $3.4(1.4)$ \\
$b_{i f}$ & $3.0(1.6)$ & $2.0(0.0)$ & $6.0(0.0)$ & $4.0(0.0)$ & $4.3(1.6)$ \\
$l_{i f}^{b}$ & $2.37(0.05)$ & $2.38(0.05)$ & $2.38(0.03)$ & $2.37(0.04)$ & $2.43(0.09)$ \\
$l_{i f}^{c}$ & $2.46(0.07)$ & $2.47(0.07)$ & $2.38(0.03)$ & $2.42(0.05)$ & $2.55(0.10)$ \\
$d$ & $\cdots$ & $2.2(0.25)$ & $3.3(0.2)$ & $3.0(0.2)$ & $2.9(0.7)$ \\
$B$ & 4.1 & 4.0 & 5.8 & $4.0-6.0$ & 4.1 \\
$\Delta E$ & 2.4 & 2.2 & 0.0 & 3.0 & 2.3 \\
\hline \hline
\end{tabular}

involving the diffusion or the creation and/or annihilation of coordination defects, this algorithm requires the addition of "ghost bonds" between pairs of atoms whose coordination goes up or down, preserving the completeness and uniqueness of the classification, but increasing significantly the complexity of the algorithm.

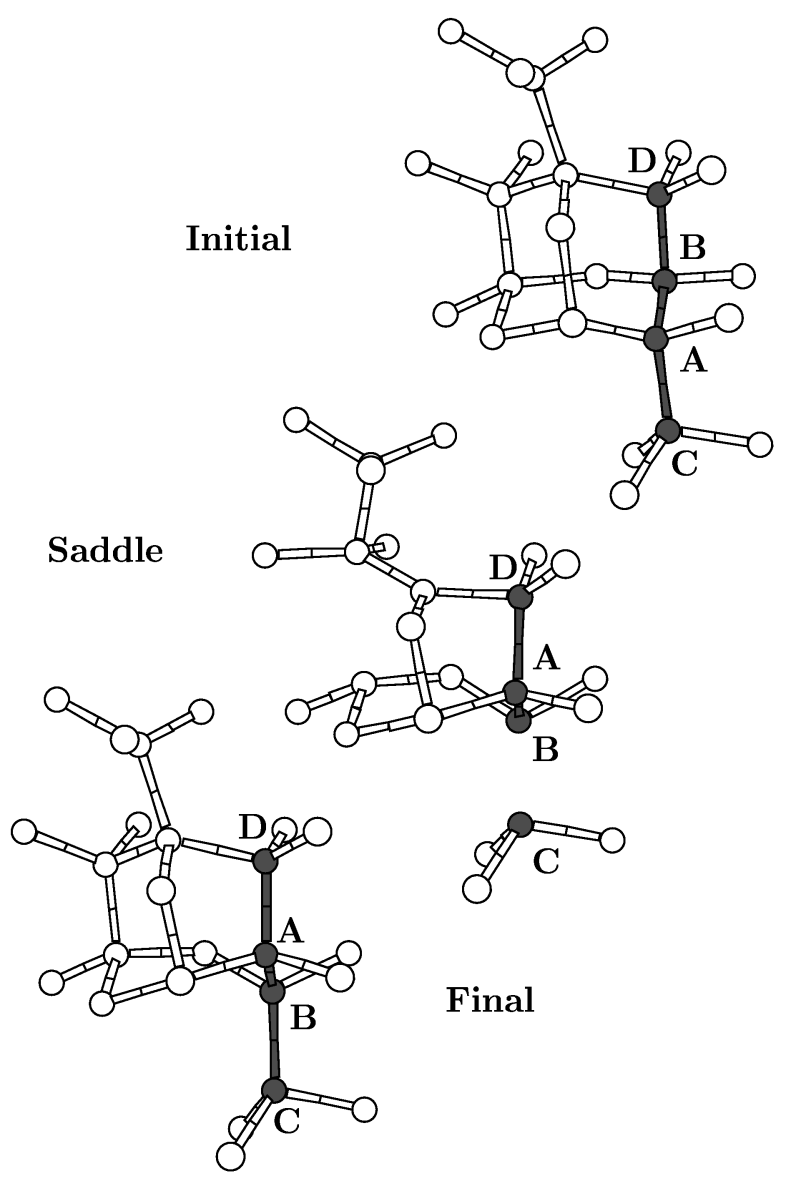

FIG. 2. Typical example of a bond rearrangement. Only four atoms, labeled "A," "B," "C," and "D," change their list of neighbors from the initial to the final state.
Applying the classification scheme discussed above to the perfect events, we find that three mechanisms account for more than $85 \%$ of the distribution. The most frequent type of event, $68 \%$ of all, is, in the notation introduced above, ABACBD and is illustrated in Figs. 2 and 3. It corresponds, surprisingly, to the bond-switching algorithm proposed by Wooten, Winer and Weaire (WWW) [13] for the preparation of amorphous silicon from the crystalline state: two bond-sharing atoms exchange a pair of neighbors. Moreover, the distribution of the energy difference $\Delta E$ for this mechanism is similar to that obtained by Wooten and Weaire [12], indicating a mostly uniform sampling of this mechanism by ART. With a peak at low energy and a relatively narrow spread of about $2 \mathrm{eV}$, the distribution of the activation energy suggests that this mechanism is the dominant one in its class.

The second most common type of perfect event (12\%), in our database, is classified as ABACBDAEBFAGBH and is illustrated in Fig. 3. These events involve a perfect switch of two nearest-neighbor atoms. Such a mechanism, dubbed concerted exchange (CE), was proposed some time ago by Pandey to explain self-diffusion in $c$-Si [14],

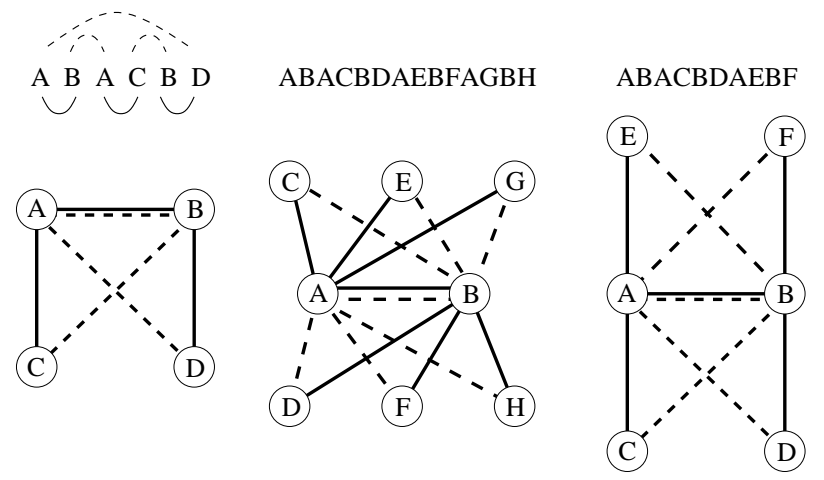

FIG. 3. Most frequent events in our simulation. The left figure corresponds to the WWW bond-switching mechanism, the middle figure to concerted exchange, and the right figure to a double bond-switching mechanism. 
and its existence is still debated. The activation energy distribution for the $\mathrm{CE}$ is strongly skewed: the lowest events happen at $3.6 \mathrm{eV}$, with a tail that runs up to about $12 \mathrm{eV}$. The lower part of the distribution is in agreement with the activation energy calculated for $c-\mathrm{Si}$ using the Stillinger-Weber potential [15] but is higher than for WWW-type events.

The CE can be decomposed in three basic WWW bondswitching steps: each atom exchanges three neighbors with the other atom. As could therefore be expected, the double bond-switching mechanism, represented in our classification as ABACBDAEBF, is also found. With a frequency of $8 \%$, the double WWW is the third most frequent mechanism (shown in Fig. 3). It displays an even wider and flatter distribution of activation energies than the $\mathrm{CE}$ mechanism, starting at about $3.5 \mathrm{eV}$ and running up to $17 \mathrm{eV}$.

These three mechanisms account for 703 out of 802 perfect events. All other mechanisms occurred less than $1 \%$ of the time and involve, typically, a larger number of bond exchanges, and a higher activation energy.

Although more than $95 \%$ of the atoms in our three samples are fourfold, most events actively involve defects either through diffusion (conserved events) or creation and/or annihilation. Topological analysis reveals that no dominant class of events emerges in either of these cases, due to a much larger number of possible topological environments that can be found when defects are around. It is nevertheless still possible to highlight some generic trends. The creation and/or annihilation mechanisms and other results from this study will be presented in a longer paper [16], but we can say a word about diffusion of defects.

Contrary to conventional experimental imagery, we do not find any significant presence of crystalline-type defects in these events, such as interstitials or vacancies. Diffusion is controlled by jumps of coordination defects that can require a significant reorganization of the lattice: the number of broken and created bonds is 1.25 bond higher than in perfect events. Again, as shown in Table I, there is no evidence of diffusion dominated by highly stretched bonds. One might also expect that defect-mediated relaxation is easier than fully fourfold coordination. In contrast to this prediction, however, we find that the distribution of activation energies is very similar to that of the perfect events, with a peak at around $4 \mathrm{eV}$ and a tail extending to $14 \mathrm{eV}$. Finally, as mentioned previously, the total number of atoms involved is found to be essentially insensitive to the class of events. This might be explained by the fact that more than $60 \%$ of conserved events have a root based on one of the three mechanisms dominating the perfect events (Fig. 3).

We have presented here the first detailed analysis of microscopic diffusion and relaxation mechanisms in $a-\mathrm{Si}$, using a topological classification of events. In particular, we find that perfect (non-defect-based) diffusion involves mainly mechanisms corresponding to variations on the bond-switching procedure of Wooten, Winer, and Weaire, which include the concerted-exchange mechanism proposed by Pandey. The size and activation energy distributions of events are realistic and provide a first check with experiment. We also find that the microscopic mechanisms responsible for defect-involved diffusion in $a-\mathrm{Si}$ differ from those in the crystal and are based on jumps of coordination defects; there is no trace of vacancy- or interstitial-mediated diffusion. In view of the generic character of the analysis, these conclusions should apply to other elemental tetrahedral amorphous semiconductors as well.

All of these mechanisms result directly from the activation-relaxation technique simulations, with no $a$ priori input provided about the nature of possible mechanisms. Much remains to be analyzed from the large database presented here. In particular, it will be necessary to recompute some of these barriers using more accurate interactions such as tight-binding or local density approximation functionals, and to analyze in more detail the nature of defect annihilation and/or creation.

N.M. acknowledges partial support by the Stichting FOM (Fundamenteel Onderzoek der Materie) under the MPR program.

*Email address: barkema@phys.uu.nl

†Permanent address.

Email address: mousseau@helios.phy.ohiou.edu

[1] T. A. Weber and F.H. Stillinger, Phys. Rev. B 32, 5402 (1985).

[2] G. Boisvert, L. J. Lewis, and A. Yelon, Phys. Rev. Lett. 75, 469 (1995).

[3] G. T. Barkema and N. Mousseau, Phys. Rev. Lett. 77, 4358 (1996).

[4] W. Frank, U. Gösele, H. Mehrer, and A. Seeger, in Diffusion in Crystalline Solids, edited by G. E. Murch and A. Nowick (Academic, New York, 1984).

[5] S. Roorda, W. C. Sinke, J. M. Poate, D. C. Jacobson, S. Dierker, B. S. Dennis, D. J. Eaglesham, F. Spaepen, and P. Fuoss, Phys. Rev. B 44, 3702 (1991).

[6] J.H. Shin and H.A. Atwater, Phys. Rev. B 48, 5964 (1993).

[7] G. Müller, G. Krötz, S. Kalbitze, and G. N. Greaves, Philos. Mag. B 69, 177 (1994).

[8] N. Mousseau and G. T. Barkema, Phys. Rev. E 57, 2419 (1998).

[9] N. Mousseau and L. J. Lewis, Phys. Rev. Lett. 78, 1484 (1997); Phys. Rev. B 56, 9461 (1997).

[10] F.H. Stillinger and T. A. Weber, Phys. Rev. B 31, 5262 (1985).

[11] P. A. Fedders, Y. Fu, and D. A. Drabold, Phys. Rev. Lett. 68, 1888 (1992); Q. Li and R. Biswas, Phys. Rev. B 52, 10705 (1995).

[12] F. Wooten and D. Weaire, Solid State Phys. 40, 1 (1987).

[13] F. Wooten, K. Winer, and D. Weaire, Phys. Rev. Lett. 54, 1392 (1985).

[14] K. C. Pandey, Phys. Rev. Lett. 57, 2287 (1986).

[15] E. Kaxiras and K.C. Pandey, Phys. Rev. B 38, 12736 (1988).

[16] N. Mousseau and G. T. Barkema (to be published). 